\title{
BIBECHANA
}

A Multidisciplinary Journal of Science, Technology and Mathematics ISSN 2091-0762 (Print), 2382-5340 (Online)

Journal homepage: http://nepjol.info/index.php/BIBECHANA

Publisher: Research Council of Science and Technology, Biratnagar, Nepal

\section{General relativity, quantum behavior and Doppler factor basics in laser-driven light sailing and solar sailing}

Gabriel James Tambunga

916 S. Delhi Street, Philadelphia, PA, U.S.A.

E-mail: mexicanengineer911@gmail.com

Article history: Received 12 May, 2016; Accepted 2 August, 2016

DOI: http://dx.doi.org/10.3126/bibechana.v14i0.15407

This work is licensed under the Creative Commons CC BY-NC License.

https://creativecommons.org/licenses/by-nc/4.0/

(c) (1) (8)

Abstract

Basic concepts of the Quantum Behavior of atoms and molecules, modified concepts of General Relativity and the Doppler Factor were applied to a reflection associated with solar sailing and laserdriven light sailing, which provides the motion in the sailing due to energy and momentum transfer of the photons to the sail. The result of this application is the energy and momentum being conserved, for the first time, from the perspective of the reflector and the source. The magnitude and the direction of the reflector, after a reflection, are not consistent with current beliefs of the reflection of a photon, which is a consequence of the conservation proposed in this paper. An experimental test to verify the actual energy and momentum transfer from a photon to a reflector is also proposed.

Keywords: General Relativity; Quantum Behavior; Doppler Factor; Laser-Driven Light Sailing; Solar Sailing.

\section{Introduction}

Quantum Electrodynamics (QED) and the Doppler Factor were previously applied to Laser-Driven Light Sailing [1], where these applications indicated the laser source used to propel the reflector needed to decrease the wavelength of the photons as the sail increased in velocity. This is because the atoms or molecules associated with the reflection would observe a larger wavelength due to the Doppler Factor when the reflectors velocity increases away from the source, where the atoms or molecules would absorb and emit photons, a reflection, at discrete energies. However, in the paper the conservation of energy from the reflectors perspective indicated the reflector should not be in motion, as the photon energy would enter and exit the reflector at the same value. That is, according to current viewpoints $[2,3]$, the 
reflector should be in motion, a kinetic energy, after a reflection, but the paper indicated the reflector should not be in motion as kinetic energy cannot be gained per the conservation of energy.

The current paper will continue the research and show how energy and momentum is conserved, from both a reflector and sources perspective, by using the Quantum Behavior of atoms and molecules and modified concepts of General Relativity (GR), while maintaining the use of the Doppler Factor. The results of this current paper will indicate changes to current beliefs of a reflection and will suggest an experimental apparatus that can used to confirm or challenge current beliefs. Only parallel vectors will be used in this paper, for simplicity. As the current paper is only an introduction of the Quantum Behavior of atoms and molecules and modified concepts of GR to a reflection, performing the suggested experiment is beyond the scope of the current paper.

\section{Emitted Energy and the Einstein Field Equations}

When a source emits a photon, the photon has an energy $h f_{s}$, a momentum $h / \lambda_{s}$, and a space-time geometry $G_{\mu v}$, where $G_{\mu \nu}$ is calculated from the energy-momentum tensor, $T_{\mu \nu}$ [4]. That is,

$$
G_{\mu \nu}=\frac{8 \pi G}{c^{4}} T_{\mu \nu}
$$

This space-time geometry would also be observed by a reflector, when both source and reflector are in the same reference frame. However, if a reflector is in motion and requires the photon to be at an energy $h f_{r}$, for a reflection, then the source would need to emit a photon energy of

$$
\frac{\sqrt{1+\beta}}{\sqrt{1-\beta}} h f_{r}
$$

due to the Doppler Factor, where $\beta$ is the velocity of the reflector from the sources perspective divided by the speed of light. As a result, per Equation (1), the space-time geometry of the photon, from the sources perspective, would also change. That is, the emitted photon from the sources perspective would have a space-time geometry of $R_{\mu v}$, and the space-time geometry from the reflectors perspective would be $G_{\mu v}$. However, for this paper, the space-time geometry observed by the reflector will be the same space-time geometry the source observes, $R_{\mu v}$, as this will be necessary, as will be shown, for balancing the conservation of energy and momentum.

\section{Reflector Physical Description}

To facilitate the discussion in this paper the physical characteristics of a reflector may be necessary. A reflector is composed of atoms and molecules, where one side of a reflector absorbs then emits photons, a reflection, in the opposite direction of the original photon. The other side of the reflector does not absorb or emit photons. 


\section{Energy and Momentum of a Non-Moving Source}

According to Quantum Theory [5], when an atom or molecule within the reflector absorbs a photon the atom or molecule is in an excited state, where the electron is at a higher energy level and is equal to the energy of the photon. For the atom or molecule to be in motion both momentum and energy must be conserved. As there is no excess energy for the atom or molecule to be in motion after absorption, then the momentum of the photon must be changed to momentum within the atom or molecule, similar to the way a photons energy changes to an electrons excited energy state within an atom or molecule. Further, the direction of the momentum, prior to absorption, does not affect the excited state after absorption. For this paper, there is no excess momentum to cause motion of the atom or molecule, as the momentum from the photon is absorbed into the atom or molecule.

After a photon is absorbed, the photon energy and momentum remains in the atom or molecule for time $\tau$, the decay time. That is; the time it takes for the atom or molecule to be in this excited state; from absorption to emission. During this time, the side of the reflector that absorbs and emits the photon is in a higher energy state, where there is a small increase of the space-time geometry according to the Einstein Field Equations. As a result, a small acceleration occurs for time $\tau$ for each atom or molecule that absorbs a photon.

\section{Energy and Momentum of a Moving Source}

If a moving source emits a photon energy as indicated in Equation (2), but the reflector observes a spacetime geometry of $R_{\mu v}$, the space-time geometry observed by the source, this would indicate to the reflector there will be more or less energy and momentum associated with the photon than what will be absorbed.

For a source moving away from a reflector, the enlarged space-time geometry, from the reflectors perspective, indicates there is an excess energy of

$$
\frac{\sqrt{1+\beta}}{\sqrt{1-\beta}} h f_{r}-h f_{r}
$$

and an excess momentum of

$$
\frac{\sqrt{1+\beta}}{\sqrt{1-\beta}} \frac{h}{\lambda_{r}}-\frac{h}{\lambda_{r}}
$$

pointing toward the reflector.

For a source moving toward a reflector the $\beta$ values would be negative, so the energy value would be less than what is required to excite the atom or molecule. From the reflectors perspective, equations (3) and (4) are still used but the energy would be negative with the momentum pointing in the direction of the incoming photon. 
As in a non-moving source, after absorption, there would be an enlarged energy state on the reflection side of the reflector for time $\tau$, resulting in an acceleration for the atoms or molecules that absorbed a photon.

\section{Emission for a Non-Moving and Moving Source}

After emission, the energy state and momentum within the atom or molecule are return to its state prior to absorption. The momentum of the photon contains a direction; however, this direction has no effect on the energy state and momentum within the atom or molecule after emission. As the emitted photon has the same energy and momentum as the excited state of the atom or molecule, there would be no excess for motion. Further, as there is no more excited state, the space-time geometry of the atom or molecule returns to the geometry prior to absorption.

After the emission, the reflector emits and observes a photon with an energy, momentum, and space-time geometry of $h f_{r}, h / \lambda_{r}$ and $G_{\mu \nu}$, respectively, where the momentum is pointing toward the source. The source would observe an energy and momentum of

$$
\frac{\sqrt{1+\beta^{\prime}}}{\sqrt{1-\beta^{\prime}}} h f_{r}
$$

and

$$
\frac{\sqrt{1+\beta^{\prime}}}{\sqrt{1-\beta^{\prime}}} \frac{h}{\lambda_{r}}
$$

respectively, where $\beta$ ' is the velocity of the reflector from the sources perspective divided by the speed of light. However, by maintaining the space-time geometry from the reflectors prespective, $G_{\mu v}$, there is more or less energy and momentum associated with the photon than what is observed. This being an energy and momentum of $h f_{r}$ and $h / \lambda_{r}$, respectively. Taking the difference of what was emitted by the source and what was observed by the source, after the reflectors emission, through $G_{\mu v}$ determination, results in an energy difference of

$$
\left(\frac{\sqrt{1+\beta}}{\sqrt{1-\beta}} h f_{r}-h f_{r}\right)
$$

and a momentum of

$$
\left(\frac{\sqrt{1+\beta}}{\sqrt{1-\beta}} \frac{h}{\lambda_{r}}-\frac{h}{\lambda_{r}}\right)
$$

This energy value and this scalar value of the momentum are the same values as the energy and momentum applied to the reflector that are not associated with what was absorbed into the atom or 
molecule, equations (3) and (4). Equations (3) and (4) apply to a moving source and a non-moving source, as the results of a non-moving source would be zero.

\section{Necessities for Energy and Momentum Conservation}

The reasoning and analysis provided in his paper shows energy and momentum as conserved. However, for the conservations to uphold the following was used and, to the author's knowledge, has not been considered in laser-driven light sailing or solar sailing.

(a) The space-time geometry associated with the photon at the source is maintained with the photon despite observers or reflectors relative motion.

(b) The energy and momentum of an absorbed photon becomes the energy and momentum within the atom or molecule, leaving no excess of energy and momentum.

(c) The direction of the photon's momentum, when absorbed in an atom or molecule, does not impact the excited state of the atom or molecule.

(d) The energy and momentum of an emitted photon originates from the energy and momentum within the atom or molecule, leaving no excess of energy and momentum.

(e) The direction of the photon's momentum, when emitted from an atom or molecule, does not impact the state of the atom or molecule after emission.

(f) Despite absorbed energy and momentum of a photon, the space-time geometry of the photon determines the excess or deficiency of the actual energy and momentum of a photon.

For (e) above, the direction of the photon is engineered, via the reflector, to emit in a certain direction. However, only the state of the careful placement of molecules, after an emission, is affected. That is, the molecule decreases to a lower state only.

\section{Discussion}

Current beliefs for motion of a reflector in laser-driven light sailing and solar sailing do not consider individual events, such as a photons energy and momentum changing to energy and momentum within an atom or molecule, and energy and momentum within an atom or molecule changing to a photons energy and momentum. By not considering the individual events, the conservation laws were not satisfied for both the source and reflector. However, by considering the individual events, as what was done in this paper, the conservation laws are satisfied between each event.

In a previous paper [1], it was determined motion due to a reflection was a result of the Doppler Shift. However, the balancing of the energy and momentum in the current paper indicates limitations in the energy of a reflector. 


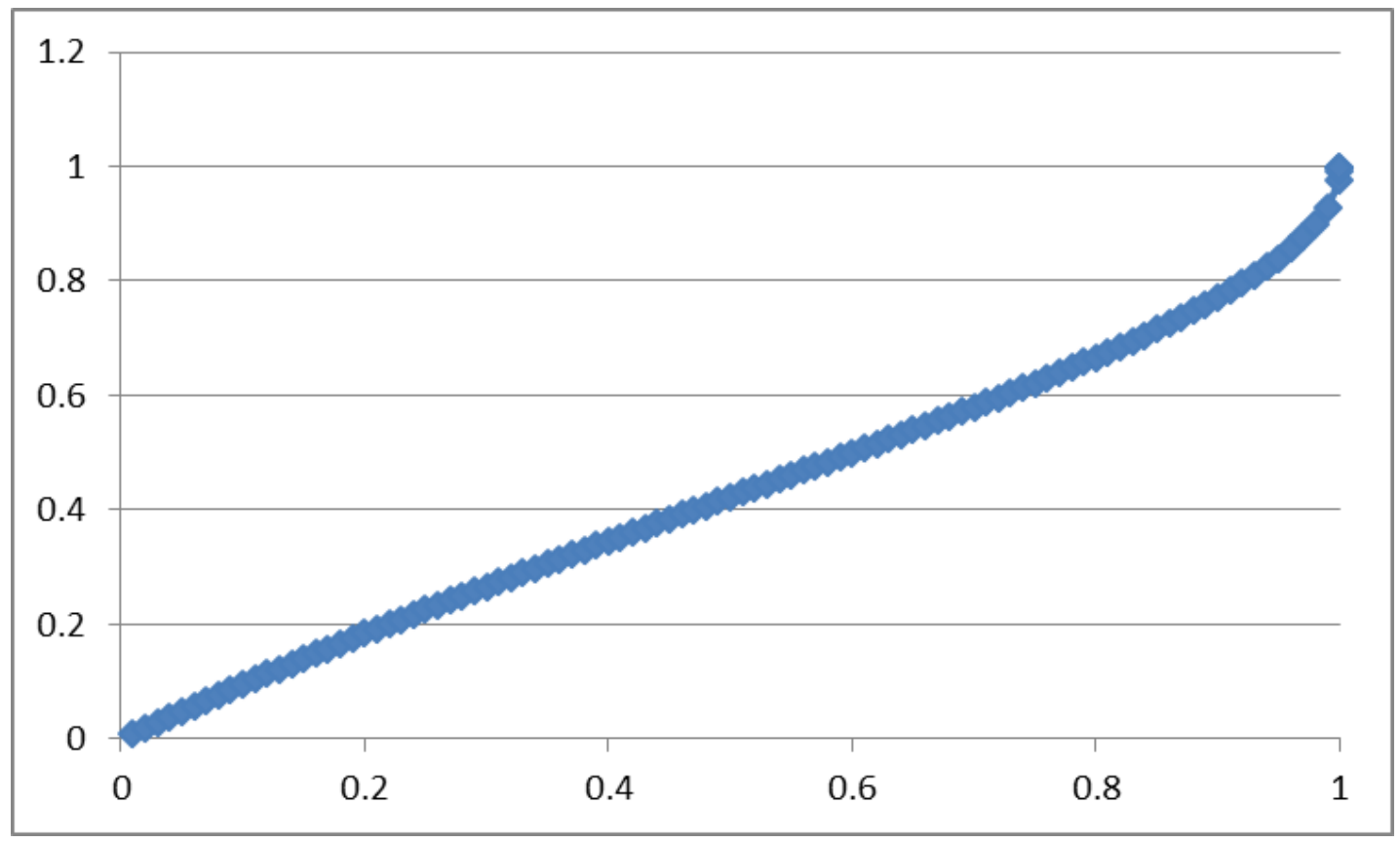

Fig. 1: A plot of $\beta$ (X-axis), and the excess energy, in $h f_{r}$, of a single atom or molecule applied to a reflector (Y-axis), when a source is moving away from a reflector. This plot indicates that the velocity of the source must be at approximately 0.8 times the speed of light for the reflector to be twice the energy of a single photon. Current beliefs indicate that twice the momentum of a photon [6,7], at minimum, will be applied to reflector despite the speed or direction of the source.

\section{A Proposed Experimental Test for Verification}

Though the conservation of energy and momentum are balanced, the direction of motion of the atom or molecule, during absorption, is inconsistent with current beliefs and practices. That is, when a source and reflector are moving towards each other, this result in a reflector increasing its velocity towards the source, according to the viewpoint presented in this paper. However, current beliefs, such as Doppler Cooling [8], indicate the atom or molecule would slow down in this same situation. A test to determine the actual direction of a reflector due to a reflection, from a moving or non-moving source, can be performed as follows:

Engineered Molecule: A molecule would need to be engineered that can align in a magnetic field in a way that will cause a photon to emit vertically, where the location on the molecule where the photon emits from will contain an unoccupied energy state in the environment where the molecule will be used, unless stimulated by the experimental setup. 
Magnetic Field: A magnetic field will cause the molecule to orbit an apparatus, where sensors to detect the molecule and photon will be above and below the path the molecule follows. The magnetic field will need to be designed to not be strong enough to maintain the molecule in the magnetic field if a vertical momentum of the molecule is above a threshold.

Source: The path of the photons from the source, which is either moving away or towards the molecule, to the molecule must be unperturbed. This is because, for example, funneling, reflecting or re-directing of the photons will cause the space-time geometry of the photons to change to the space-time geometry of a non-moving source. This will cause the molecule to not move from the magnetic field.

Further, this experimental setup may also determine the effect of the increased space-time geometry on the molecule for a non-moving source during the time the photons energy and momentum are in the molecule. This can be done by decreasing the threshold that holds the molecule in the magnetic field.

\section{References}

[1] G. J. Tambunga, International Letters of Chemistry, Physics and Astronomy, 19 (2013) 10-14.

[2] G. Vulpetti, L. Johnson, G. L. Matlof, Solar Sails: A Novel Approach to Interplanetary Travel, second ed., Springer-Praxis, Chichester, UK, (2015).

http://dx.doi.org/10.1007/978-1-4939-0941-4

[3] Y. K. Bae, Physics Procedia, 38 (2012) 253-279. http://dx.doi.org/10.1016/j.phpro.2012.08.026

[4] C. W. Misner, K. S. Thorne, J. A. Wheeler, Gravitation, W. H. Freeman and Company, New York, (1973).

[5] Information on http://www.feynmanlectures.caltech.edu/III_02.html

[6] M. Guthrie, Solar Sails - A Discussion ( 2011). https://web2.ph.utexas.edu/ mwguthrie/p.solarsails.pdf

[7] Information on http://www.feynmanlectures.caltech.edu/I_39.html

[8] D. J. Wineland, W. M. Itano, Physical Review A, 20 (1979) 1521-1540. http://dx.doi.org/10.1103/PhysRevA.20.1521 